importance of spatial context increases over the study period. No analogous pattern is visible for non-COVID mortality. Higher relative deprivation is associated with increased COVID-19 mortality at all stages of the pandemic but does not explain structural inequalities.

Conclusion Results support initial stochastic viral introduction in the South, with initially high inequality decreasing before the establishment of regional trends by June and July, prior to reported regionality of the 'second-wave'. We outline how this framework can help identify structural factors driving such processes, and offer suggestions for a long-term, locally-targeted model of pandemic relief in tandem with regional support to buffer the social context of the area.

\section{P48 RISK OF COVID-19 INFECTION AMONG MEN AND WOMEN DURING THE LOCKDOWN OF SPRING 2020 IN FRANCE}

${ }^{1}$ Lola Neufcourt*, ${ }^{1}$ Camille Joannès, ${ }^{1}$ Marine Maurel, ${ }^{1}$ Niamh $M$ Redmond, ${ }^{1}$ Cyrille Delpierre, ${ }^{1,2}$ Michelle Kelly-Irving. ' CERPOP, University, de Toulouse, Inserm, UPS, Toulouse, France, ${ }^{2}$ Iferiss-Fed 4241, University, Toulouse III Paul Sabatier, Toulouse, France

\subsection{6/jech-2021-SSMabstracts. 136}

Background In the context of the Covid-19 pandemic, several factors such as age, chronic disease or obesity have been associated with adverse outcomes and mortality from Covid-19. However, the social distribution of Covid-19 infection among men and women was largely neglected in France, mainly due to a lack of data. The aim of this study is to describe and analyse the risk of Covid-19 infection in relation to sex, and the influence of other social factors, specifically occupation, in this association.

Methods We used data from the citizen science initiative 'Baromètre Covid-19'. Each week, an internet survey was administered to a sample of 5,000 people representative of the French mainland population aged 18 and over, using the quota method. A total of 25,001 participants were interviewed between 7 April and 11 May 2020. We used multivariable nested logistic regression modelling to study the relationship between sex, occupation and Covid-19 infection. Confounders included age, region of residence, population density, whether you worked outside of home during the lockdown, house overcrowding, comorbidities and body mass index.

Results Women reported a medical diagnosis of Covid-19 infection more often than men (4\% vs. $3.2 \%)$. In a model adjusted for confounders, women were $23 \%$ more likely to report a medical diagnosis of Covid-19 infection than men $(\mathrm{OR}=1.23$ [95\%-CI=1.06-1.42]). Controlling for sex and socioeconomic variables (occupation), the risk of infection for women was reversed $(\mathrm{OR}=0.84[95 \%-\mathrm{CI}=0.59-1.19])$. While most men, other than executives, were less likely to report the infection, this association was not observed amongst women.

Conclusion Occupation was found to influence the relationship between sex and Covid-19 infection suggesting a gender effect. The differences in the risk of infection between men and women require exploration with regard to socioeconomic factors. The social roles of women and men are associated with a non-random distribution of the virus, potentially reflecting structural societal inequalities.

\section{P49 COVID-19 AT THE DEEP END: EXPERIENCES OF PRIMARY CARE STAFF WORKING IN THE MOST DEPRIVED AREAS OF ENGLAND DURING THE COVID-19 PANDEMIC}

Claire Norman*, Josephine M Wildman, Sarah Sowden. Population Health Sciences Institute, Newcastle University, Newcastle upon Tyne, UK

\subsection{6/jech-2021-SSMabstracts. 137}

Background COVID-19 is disproportionately impacting people in low-income communities. Primary care staff in areas of high blanket deprivation (also known as the 'Deep End') have unique insights into the challenges posed by the pandemic. We aim to explore the impact of the COVID-19 pandemic from the perspective of Deep End primary care practitioners in North East England, the most deprived region of the country.

Methods Semi-structured interviews followed by thematic analysis. 13 participants were interviewed (11 GPs, 1 nurse practitioner and 1 district nurse) with Deep End careers ranging from 3 months to 31 years. Participants were recruited via purposive and snowball sampling. Semi-structured interviews were conducted using video-conferencing software. Data were analysed using thematic content analysis. Participants were interviewed between September-December 2020, at the start of the UK second wave of the COVID-19 pandemic.

Results Our results can be categorised into three broad themes: the immediate health risks of COVID-19 on Deep End general practices and patients; factors likely to exacerbate the effects of existing socioeconomic deprivation; and wider implications for remote consulting.

Discussion Deep End practitioners have valuable insights into the impact of social distancing restrictions and remote consulting on patients' health and wellbeing and on the delivery of primary care in areas of deep deprivation. Their experiences should guide future pandemic response measures and any move to 'digital first' primary care to ensure that existing inequalities are not worsened.

\section{P50 TIME-VARYING SELECTION BIAS IN ANALYSES OF COVID-19 IN UK BIOBANK}

Alice R Carter*, Gareth J Griffith, Apostolos Gkatzionis, Rachael Hughes, George Davey Smith, Deborah Lawlor, Kate Tilling. Medical Research Council Integrative Epidemiology Unit, Population Health Sciences, University of Bristol, Bristol, UK

\subsection{6/jech-2021-SSMabstracts. 138}

Rationale Associations between COVID-19 risk factors and COVID-19 outcomes change over time, likely due to selection into who receives a COVID-19 test. When studies do not account for the changes in testing criteria, the association between a risk factor and outcome is a joint estimate across time. The transportability of a joint estimate aggregated over multiple testing periods may be limited. To improve generalisability, it is desirable to estimate effects net of time-varying selection.

Aim 1) Demonstrate variation in the association between covariates expected to associate with testing, and those which would not, on COVID-19 at different timepoints. 2) Apply methods to mitigate biases in empirical estimates.

Methods Analyses will be carried out on up to 421,037 UK Biobank participants residing in England at baseline (mean 
age at baseline $=56 ; 55 \%$ female). Risk factors will be determined at baseline (from 2006 to 2010), and COVID-19 outcomes will be ascertained from linked Public Health England COVID-19 test data and mortality statistics.Univariate cox proportional hazard models will be used to explore how associations between time-varying and time-stable variables change over time with; i) having a test for COVID-19, ii) testing positive for COVID-19 and iii) dying with COVID19. Time-varying risk factors will be based on measures of socioeconomic position (SEP) including education, Townsend deprivation index and income. $\mathrm{ABO}$ blood group will be considered as a time-stable risk factor. Distinct time periods will be defined based on changes in testing definitions and changes in lockdown restrictions. Inverse probability weights will then be calculated for each time period. These weights will then be applied to models estimating risk across all time periods.

Expected Results Preliminary analyses show that the size of the association between SEP and i) COVID-19 testing and ii) testing positive for COVID-19, changes across the course of the pandemic. These differences may be due to differential testing and not time-varying causal effects of the risk factor. We expect inverse probability weights will provide estimates closer to the true value for the association between each risk factor and outcome, independent of selection pressures on receiving a COVID-19 test. Population Health Relevance. Where studies do not account for timevarying selection pressures, the causal interpretations and the validity of results may be distorted. Where these findings are to be translated into developing population level or pharmaceutical interventions to mitigate against COVID-19 outcomes, efforts may be diverted away from more important risk factors.

\section{P51 A SURVEY OF THE IMPACT OF WAVE ONE OF THE COVID-19 PANDEMIC ON PARTICIPANTS OF THE HERTFORDSHIRE COHORT STUDY}

Gregorio Bevilacqua, Alice Paul, Ilse Bloom*, Karen Jameson, Jean Zhang, Cyrus Cooper, Kate Ward, Elaine Dennison. MRC Lifecourse Epidemiology Unit, University of Southampton, Southampton, UK

\subsection{6/jech-2021-SSMabstracts. 139}

Background Covid-19, a coronavirus that originated in China in late 2019, spread globally to be declared a pandemic by the World Health Organisation in March 2020. The aim of this study was to usean existing cohort of community-dwelling older adults, the Hertfordshire Cohort Study (HCS), to understand how wave one of the Covid-19 pandemic impacted UK older adults, a group particularly vulnerable to severe disease.

Methods 71 eligible participants, 39 males and 32 females (drawn from the HCS study, mean age 83.6 (2.5) years, all Caucasian, and community dwelling) were contacted by telephone and asked to complete a questionnaire administered by a trained researcher. Data collection occurred over the period of July to October 2020.

Results Over a third (37.1\%) of respondents lived alone. Of the remainder, $86.4 \%$ lived with a spouse while the remaining $13.6 \%$ lived with family. Of concern, $19.7 \%$ of participants had felt they needed to go out despite not wanting to; $47.1 \%$ had heard of the NHS Volunteer Responders programme, although only $3(4.2 \%)$ had made use of this or other support services. Almost a third (31\%) of participants reported they had access to a smartphone, while $62 \%$ reported having unlimited internet access, usually using a tablet or computer. Over two thirds (69.0\%) of participants rated their understanding of Covid-19 itself as good. Perhaps unsurprisingly, a large majority $(88.7 \%)$ of participants said their life was different compared to before Covid-19; $80.3 \%$ had less social contact and more than half (52\%) of respondents reported being less physically active than before the pandemic (and only $4 \%$ more so). However, levels of sleep, alcohol consumption and diet were reportedly generally unchanged over the timeframe surveyed.

Conclusion We have reported the experience of the first wave of the Covid-19 pandemic among participants of an older Caucasian community-dwelling UK cohort, highlighting the need to consider this group when developing public health interventions to support health and wellbeing, including those employing smartphone technology. Further larger studies in groups of wider socioeconomic status and more diverse racial background are indicated.

\section{P52 1 THE EXPERIENCES OF COMMUNITY-DWELLING OLDER ADULTS DURING THE COVID-19 PANDEMIC: PRELIMINARY FINDINGS FROM A QUALITATIVE STUDY WITH HERTFORDSHIRE COHORT STUDY PARTICIPANTS}

\footnotetext{
1,2 Ilse Bloom*, 1,2 Jean Zhang, 'Gregorio Bevilacqua, 1,2Wendy Lawrence, 'Kate Ward ${ }^{1}$ Cyrus Cooper, ${ }^{1}$ Elaine Dennison. ${ }^{1} M R C$ Lifecourse Epidemiology Unit, University of Southampton, Southampton, UK; ${ }^{2}$ NIHR Southampton Biomedical Research Centre, University of Southampton and University Hospital Southampton NHS Foundation, Southampton, UK
}

\subsection{6/jech-2021-SSMabstracts. 140}

Background The COVID-19 pandemic has led to dramatic changes in people's lives globally. Older adults have been especially vulnerable to adverse effects from the pandemic including higher mortality and more severe disease complications. At the same time, social isolation, malnutrition and physical inactivity are serious concerns among older adults. The pandemic and associated restrictions may serve to exacerbate these issues, presenting increased risks to physical and mental health. The aim of this qualitative study was to explore how community-living older people in the UK experienced the first wave of the COVID-19 pandemic and how it impacted their health and well-being, and associated behaviours.

Methods Qualitative data were collected by conducting serial telephone interviews, with an interval of approximately three months. Participants were from the Hertfordshire Cohort Study, all aged over 80 years. Discussions were audiorecorded, information related to the COVID-19 pandemic was transcribed verbatim and transcripts analysed thematically. Interviews were conducted from March to October 2020.

Results Twelve participants were included in the study (7 men and 5 women). Data from a total of 35 qualitative interviews were used, comprising two or three timepoints per participant. Preliminary analysis identified five overarching themes: 1) shopping strategies and food accessibility, 2) limitations on activities and going out, 3) disruption to healthcare, 4) social and psychological repercussions, and 5) coping strategies. Initial findings highlight challenges associated with accessing 